No. $97-20$

\title{
Type la Supernovae: Their Origin and Possible Applications in Cosmology
}

Ken'ichi Nomoto, Koichi Iwamoto, Nobuhiro Kishimoto

Department of Astronomy, University of Tokyo, Bunkyo-ku, Tokyo 113, Japan

To appear in Science, 1997, May 30 
The University of Tokyo, Astronomy Preprints

\title{
Type la Supernovae: Their Origin and Possible Applications in Cosmology
}

\author{
Ken'ichi Nomoto, Koichi Iwamoto, Nobuhiro Kishimoto \\ Department of Astronomy, University of Tokyo, Bunkyo-ku, Tokyo 113, Japan
}

\begin{abstract}
Spectroscopic and photometric evidence indicates that Type Ia supernovae (SNe Ia) are the thermonuclear explosions of accreting white dwarfs. However, the progenitor binary systems and hydrodynamical models for SNe Ia are still controversial. The relatively uniform light curves and spectral evolution of $\mathrm{SNe}$ Ia have led to their use as a standard candle for determining cosmological parameters, such as the Hubble constant, the density parameter, and the cosmological constant. Recent progress includes the calibration of the absolute maximum brightness of SNe Ia with the Hubble Space Telescope, the reduction of the dispersion in the Hubble diagram through the use of the relation between the light curve shape and the maximum brightness of SNe Ia, and the discovery of many SNe Ia with high red shifts.
\end{abstract}

Supernovae are classified spectroscopically as Type I if they have no hydrogen lines in their optical spectra and Type II if they have hydrogen lines in their optical spectra. Type I supernovae (SNe I) are further subclassified into types $\mathrm{Ia}, \mathrm{Ib}$, and Ic on the basis of spectra observed early in their explosion (early-time spectra) (11). $\mathrm{SNe}$ Ia are characterized by the presence of a deep Si II absorption line near wavelength $6150 \AA$ (Fig. 1), and their late-time spectra are dominated by strong blends of $\mathrm{Fe}$ emission lines. SNe Ib and Ic, in contrast, do not show this Si line. Moderately strong He I lines, especially at $5876 \AA$, distinguish $\mathrm{SNe} \mathrm{Ib}$ from SNe Ic in early-time spectra; that is, SNe Ib exhibit absorption lines of He I, whereas these lines are weak or absent in SNe Ic (11). SNe II, Ib, and Ic are now generally thought to result from the explosion of massive stars - SNe II from single stars and $\mathrm{SNe} \mathrm{Ib}$ and Ic from binary stars. There are spectroscopic and photometric indications that $\mathrm{SNe}$ Ia originate from white dwarfs that are composed of $\mathrm{C}+$ $\mathrm{O}$ with strongly degenerate electrons and have accreted sufficient mass from a companion to trigger an explosion.

However, the progenitor systems and hydrodynamical models for SNe Ia are still controversial. Many issues need to be resolved including (i) double degenerate(DD) versus single degenerate(SD) scenarios, that is, whether the companion of the white dwarf is also an electron-degenerate white dwarf or is a non-degenerate [main sequence (MS) or evolved red giant] star; (ii) Chandrasekhar mass (Ch) (2) versus sub-Chandrasekhar mass (sub-Ch) models; and (iii) the explosion mechanism in the $\mathrm{Ch}$ models. The answers to these questions could lead to improved understanding of the complicated evolution of close binaries as well as the physics of thermonuclear explosion (3). The relatively uniform light curves and spectral evolution of SNe Ia have led to their use as a standard candle to determine the Hubble constant $\left(H_{0}\right)$, the density parameter $\left(\Omega_{\mathrm{M}}\right)$, and the cosmological constant $\left(\Omega_{\Lambda}\right)$ (4). SNe Ia are important standard candles because they are bright enough to be observed out to a redshift $(z)$ of $\sim 1$. Variations of light curves and spectra among SNe Ia have recently received attention. SNe Ia with higher maximum brightnesses tend to show a slower decline in their light curves (5, 6; Fig. 2). This review summarizes our current understanding of SNe Ia and focuses on these controversial issues.

\section{Progenitors}

White dwarfs composed of $\mathrm{C}+\mathrm{O}$ are formed from intermediate mass stars $\left(M<8 M_{\odot}\right.$, where $M_{\odot}$ is the mass of our sun), undergo cooling, and eventually become dark matter, as they evolve towards fainter luminosities. In a close binary system the white dwarf evolves differently because the companion star expands to transfer matter to the white dwarf; the accreting white dwarfs are rejuvenated and in certain cases undergo thermonuclear explosions to give rise to $\mathrm{SNe}$ Ia. Theoretically the $\mathrm{Ch}$ white dwarf models and the sub-Ch models have been considered to explain the origin of SNe Ia (7). Various evolutionary scenarios have been proposed, including (i) merging of double $\mathrm{C}+\mathrm{O}$ white dwarfs with a combined mass exceeding the Ch limit (a DD scenario) (8) and (ii) accretion of $\mathrm{H}$ or $\mathrm{He}$ by mass transfer from a binary companion at a relatively high rate (an SD scenario) (17, 9).

The DD-Ch scenario is favored from the theoretical estimate of frequencies of the occurrence of SNe Ia. This has stimulated a search for DD systems, but few such systems have been discovered, and their combined mass is smaller than Ch (7). For an SD scenario, the accret- 
ing white dwarf undergoes $H$ burning near the surface, which increases or decreases the white dwarf mass depending mainly on the accretion rate, $\dot{M}$ (9). Ch mass white dwarfs can be formed with a relatively high accretion rate such as $\dot{M} \approx 10^{-8}$ to $10^{-6} M_{\odot}$ year $^{-1}$ because of relatively small mass ejection after $\mathrm{H}$ or $\mathrm{He}$ shell burning. Recently, it was found that this range of $\dot{M}$ can be extended to a rate faster than $10^{-6} M_{\odot}$ year $^{-1}$ (10). With such a high rate, the white dwarf undergoes wind mass loss without expanding its radius, thereby increasing its mass by steady $\mathrm{H}$ burning. The SD-sub-Ch scenario - that is, the explosion of sub-Ch white dwarfsis a possible outcome of accretion with $4 \times 10^{-8} M_{\odot}$ $\mathrm{yr}^{-1} \gtrsim \dot{M} \gtrsim 10^{-9} M_{\odot}$ year $^{-1}$. In this case, the ignited He shell flash is strong enough to initiate an off-center He detonation (11, 12), which induces various types of explosions.

Promising candidates for the white dwarf's companion stars for the SD scenario are (i) stars with MS masses of 1 to $1.5 M_{\odot}$, which fill the Roche lobe when they evolve through red-giants, and (ii) the 2 to $3 M_{\odot}$ stars, which undergo mass transfer near the MS. These cases of relatively fast accretion onto the white dwarf can correspond to super-soft x-ray sources (13), symbiotic stars (7), or both.

To discriminate among the SD-sub-Ch, SD-Ch, and DD-Ch scenarios, we used photometric and spectroscopic diagnostics. If any $\mathrm{H}$ or $\mathrm{He}$ is detected, the DD model could be ruled out. Although high-velocity $\mathrm{H}$ has not been observed in any SNe Ia, the upper limit to the $\mathrm{H}$ abundance $\left(\sim 10^{-4} \quad M_{\odot}\right)$ is still too high to rule out the SD models. The H-rich materials in the companion star may be engulfed in the exploding material; thus the detection of low-velocity $\mathrm{H}$ could be critical (14).

\section{Explosion Models}

Once explosive nuclear burning is ignited, it induces thermonuclear explosion of the white dwarf. The outcome of this explosion depends on how the nuclear flames and/or shock waves propagate for the $\mathrm{Ch}$ and sub-Ch mass white dwarfs. The physics involved in these processes is rather complex, and multi-dimensional simulations have been conducted to understand these processes (15). The results are still preliminary, and so we focus here on those models that can account for the basic features of SNe Ia, namely, an explosion energy of $\sim 10^{44} \mathrm{~J}$, the synthesis of a large amount of ${ }^{56} \mathrm{Ni}\left(0.4\right.$ to $\left.1 M_{\odot}\right)$, and the production of a substantial amount of intermediate-mass elements at expansion velocities of $\sim 10,000 \mathrm{~km} \mathrm{~s}^{-1}$ near the maximum brightness of the $\mathrm{SN}$ explosion.

Chandrasekhar mass models: Carbon is ignited in the central region of the white dwarf when the central density exceeds $\sim 10^{9} \mathrm{~g} \mathrm{~cm}^{-3}$. Because of strong electron degeneracy, $\mathrm{C}$ burning is so explosive that it incinerates the material into Fe peak elements (16). Afterwards, the explosive nuclear flame propagates outwards. The flame front is subject to various types of instabilities, including thermal instabilities, the Landau-Darrius instability, the Rayleigh-Taylor instability, and the Kelvin-Helmholtz instability (17). The flame speed depends on the development of these instabilities and the resulting turbulence, requiring further extensive simulations on large and small scales as well as suitable modeling of the turbulence. Behind the flame front, materials undergo explosive nuclear burning of $\mathrm{Si}, \mathrm{O}, \mathrm{Ne}$, and $\mathrm{C}$. The nucleosynthesis products depend mainly on peak temperatures, which in turn depend on the densities encountered by the flame. For densities from $10^{10}$ to $10^{6} \mathrm{~g} \mathrm{~cm}^{-3}$, the products range from Fe-peak elements (mostly ${ }^{56} \mathrm{Ni}$ ) to intermediatemass elements (Ca, Ar, S, and $\mathrm{Si}$ ), O-Ne-Mg, and $\mathrm{C}+\mathrm{O}$.

During the subsonic propagation of the deflagration wave, the densities of the whole white dwarf are decreasing because of expansion. Therefore, the densities encountered by the flame are determined by its speed, which is still uncertain. Several plausible models have been presented with one-dimensional codes $(11,12)$. In carbon deflagration models, such as model W7 (18), the average flame speed is as high as one-fifth of the sound speed. A sequence of nucleosynthesis reactions produces ${ }^{56} \mathrm{Ni}, \mathrm{Ca}-\mathrm{S}-\mathrm{Si}, \mathrm{O}-\mathrm{Ne}-\mathrm{Mg}$, and $\mathrm{C}+\mathrm{O}$ behind the deflagration wave. Because of fast propagation of the flame, the transition to the detonation could be induced by shock compression in the outer low density layers, which would produce some variations of ${ }^{56} \mathrm{Ni}$ mass and distribution, as seen in peculiar SNe Ia such as SN1991T (Fig.1). In contrast, delayed detonation models assume that the early propagation of the deflagration is as slow as a few percent of the sound speed, producing $\sim 0.1 M_{\odot}$ of ${ }^{56} \mathrm{Ni}$, and hence the transition from deflagration to detonation could occur at densities of $1 \times 10^{7}$ to $3 \times 10^{7} \mathrm{~g} \mathrm{~cm}^{-3}$. In this case, the bulk of the white dwarf has expanded to lower densities so that the detonation wave synthesizes Fe-peak elements and intermediate mass elements (19). In the pulsating delayed detonation model, the transition to detonation is assumed to occur near the maximum compression (20). Possible variations of the transition density could produce variations of the ${ }^{56} \mathrm{Ni}$ mass produced.

Sub-Chandrasekhar mass models: A central C detonation can be initiated by a He detonation-induced shock wave $(12,21$. Merging of double white dwarfs could ignite a central $\mathrm{C}$ detonation (22). If the white dwarf mass is less than $1.07 M_{\odot}$, the densities of the white dwarf matter encountered by the detonation wave can be suitable to produce sufficient amounts of ${ }^{56} \mathrm{Ni}$ and $\mathrm{Si}-\mathrm{Ca}-\mathrm{O}$. Variations of the white dwarf mass could cause the variations of the light curves of the resultant SN Ia.

Constraints on still uncertain parameters in these mod- 
els, such as the central ignition density, the flame speed, and the deflagration-detonation transition density, can be provided by comparisons of theoretical spectra and light curves with observations and by comparisons of nucleosynthesis consequences with solar isotopic ratios. A combination of processes from various models may be responsible for the variations in characteristics of the observed SNe Ia.

\section{Spectra and Light Curves}

Spectra: Spectra of SNe Ia are generally homogeneous but show some important variations. Because SNe Ia do not have a thick H-rich envelope, elements newly synthesized during the explosion can be observed in the spectra. Thus, comparison between the synthetic spectra and observations is a powerful diagnostic of the dynamics and nucleosynthesis suggested by the models. Non-local thermodynamical equilibrium(LTE) spectra have been calculated and can be used to compare with observations. A typical example (Fig. 3) shows agreement between the $\mathrm{C}$ deflagration model (unmixed W7) and the observed optical spectra of SNe 1992A and 1994D (23). The material velocity at the stellar surface near maximum light is $\sim$ $10,000 \mathrm{~km} \mathrm{~s}^{-1}$ and the spectral features are identified as those of $\mathrm{Fe}, \mathrm{Ca}, \mathrm{S}, \mathrm{Si}, \mathrm{Mg}$, and $\mathrm{O}$. This implies that the abundance distribution in the velocity space may be similar to W7. The synthetic spectra for the sub-Ch models seem to be less satisfactory $(23,24)$.

Regarding the heterogeneity in the spectra, SNe 1991T (Fig. 1) and 1991bg are the two extreme examples that have clearly revealed the presence of spectroscopically peculiar SNe Ia (25). The pre-maximum light spectra of SNe Ia show a significant variation of the composition and expansion velocities of the outermost layers, whereas the post-maximum spectra are relatively uniform except for SN 1991bg. The different ${ }^{56} \mathrm{Ni}$ mass may produce the variation of the spectra (26). Further analysis could provide the abundance distribution of various elements (Fe, Ca, $\mathrm{Si}, \mathrm{Mg}, \mathrm{O}, \mathrm{C}$, and others) in velocity space (27). Light curves: Recent high-quality charge-coupled device (CCD) observations of SNe Ia have, on one hand, supported the basic homogeneity of the optical light curve shape of SNe Ia. Good examples include SNe 1980N and 1981D in the galaxy NGC 1316, whose light curve shapes and brightnesses were almost identical. On the other hand, the observations have established significant variations of the maximum brightness, the light curve shape, and their correlation (Fig.2). In theoretical models, the explosion energy goes into the kinetic energy of expansion, $E$. The light curves are powered by the radioactive decay sequence ${ }^{56} \mathrm{Ni} \rightarrow{ }^{56} \mathrm{Co} \rightarrow{ }^{56} \mathrm{Fe}$. The calculated light curve reaches its peak at about 15 to 20 days after the explosion and declines because of the in- creasing transparency of the ejecta to gamma rays as well as the decreasing input of radioactivity. The light curve shape depends mainly on the effective diffusion time $\tau_{m} \propto\left(\kappa M / v_{\exp } c\right)^{1 / 2}$, where $\kappa$ is opacity, $c$ is the speed of light, and $v_{\exp } \propto(E / M)^{1 / 2}(15)$. For longer $\tau_{m}$, the decline of the light curve is slower. For larger $M_{\mathrm{Ni}}$, the brightness is higher. For the Ch models, typical values of $M_{\mathrm{Ni}}$ and $E$ are similar to those of W7, that is, $M_{\mathrm{Ni}}$ $=0.6 \pm 0.1 M_{\odot}$ and $E=1.3 \pm 0.1 \times 10^{44} \mathrm{~J}$.

The observed uniformity in the light curve implies a uniformity of $M, E$, and $M_{\mathrm{Ni}}$. A homogeneous $M$ and $E$ can be naturally accounted for with the Ch models, although the central density can vary with a small difference of mass near the Ch limit. For the sub-Ch models, the accretion process must somehow choose a relatively narrow mass range, such as $M \approx 1.0$ to $1.1 M_{\odot}$. The reported variation of the maximum brightness of SNe Ia (5, 6) may be a result of the variations of the ${ }^{56} \mathrm{Ni}$ mass. The amount of ${ }^{56} \mathrm{Ni}$ depends on (i) the flame speed in the fast-deflagration models, (ii) the location and density of the transition to a detonation in the delayed or late detonation models, and (iii) the white dwarf mass in the sub-Ch models. Because of variations of these parameters, a variation of the ${ }^{56} \mathrm{Ni}$ mass may be possible. For the Ch models, the dependence of optical opacities on the temperature is important (28). A smaller mass of ${ }^{56} \mathrm{Ni}$ means less heating of the surface layer, thus lowering $\kappa$ and $\tau_{m}$. This effect might explain the relation between brightness and the decline rate of the light curve. For the sub-Ch models, $M_{\mathrm{Ni}}$ varies approximately in proportion to $M$. Then for larger $M, M_{\mathrm{Ni}}$ is larger and $\tau_{m}$ is longer; in other words, brighter SNe Ia tend to decline more slowly (15).

\section{Nucleosynthesis in Galactic Chemical Evolution}

Supernovae of different types have different progenitors, thus producing different heavy elements on different time scales during the chemical evolution of galaxies. A reasonable mixture of the heavy-element yields from $\mathrm{SNe}$ Ia and SNe II should be able to explain the solar abundance pattern of heavy elements from $\mathrm{O}$ to the Fe group. Nucleosynthesis products of SNe Ia and SNe II can be combined with various ratios and compared with solar abundances of heavy elements and their isotopes. If the nucleosynthesis products of SNe II as a function of stellar masses (29) are adopted with an upper mass limit of 50 $M_{\odot}$ and the nucleosynthesis products of SNe Ia are those from model W7, the best fit to known solar abundances is obtained if the number of SNe Ia that have occurred relative to $\mathrm{SNe}$ II is $N_{\mathrm{Ia}} / N_{\mathrm{II}}=0.12$ (30) (Fig. 4). This is consistent with observation-based estimates that the SNe Ia frequency is as low as $10 \%$ of total supernova 
occurrence (31).

With this relative frequency, ${ }^{56} \mathrm{Fe}$ from $\mathrm{SNe}$ Ia is about $50 \%$ of total ${ }^{56} \mathrm{Fe}$ from all SNe. For the Ch models, the abundance ratios between neutron-rich species and ${ }^{56} \mathrm{Fe}$ provide an important constraints on the progenitor system. The central density of the white dwarf at thermonuclear runaway must be as low as $\lesssim 2 \times 10^{9} \mathrm{~g} \mathrm{~cm}^{-3}$ to avoid overabundances of ${ }^{58} \mathrm{Ni},{ }^{54} \mathrm{Cr}$, and ${ }^{50} \mathrm{Ti}$ relative to ${ }^{56} \mathrm{Fe}$, although the exact density depends on the flame speed (30). Such a low central density can be realized by the accretion as fast as $\dot{M}>1 \times 10^{-7} M_{\odot}$ year $^{-1}$, which is consistent with the SD-Ch scenario. SNe Ia yields thus contribute Fe enrichment to the chemical evolution of the galaxies. Because the progenitors of SNe Ia are low-mass stars with relatively long lifetimes, Fe could be used as a clock to look back into the timing of galaxy formation (32).

\section{Cosmological Parameters}

Hubble constant: SNe Ia are very good, but not perfect standard candles. The accuracy of the determination of $H_{0}$ using SNe Ia depends on (i) whether the peak absolute-magnitude dispersion of $\mathrm{SNe}$ Ia is sufficiently small, and (ii) whether a precise absolute-magnitude calibration of a SN Ia can be made. Recent progress has been made in the empirical determination of $H_{0}$. For the dispersion, the correlation between the maximum brightness and the decline rate (or the light curve shape) is taken into account. Multicolor light-curve shapes are also used for further corrections (6). This reduces the dispersion from 0.4 to 0.2 magnitudes in the Hubble diagram for SNe Ia (6, 33) (Fig.5). For the calibration, the distances to several host galaxies of SNe Ia (SNe 1895B, 1937C, $1960 \mathrm{~F}, 1972 \mathrm{E}, 1981 \mathrm{~B}, 1989 \mathrm{~B}$, and 1990N) have been determined from Hubble Space Telescope observations of Cepheids in these galaxies (34). On the basis of these data, $H_{0}$ is estimated to be $58{ }_{-8}^{+7}$ (34), $63.1 \pm 6.3$ (33), $64 \pm 6$ (6), and $55 \pm 3$ (35) $\mathrm{km} \mathrm{s}^{-1} \mathrm{Mpc}^{-1}$.

Successful theoretical models can, in principle, give the absolute maximum luminosities, thus providing good estimates of $H_{0}$ and $\Omega_{\mathrm{M}}$. The maximum luminosity $L_{\max }$ of SNe Ia when explained with radioactive decay models has been used to estimate $H_{0}(36)$. From a comparison with individual SNe Ia, $H_{0}=67 \pm 9 \mathrm{~km} \mathrm{~s}^{-1} \mathrm{Mpc}^{-1}$ (24), and $59 \pm 13 \mathrm{~km} \mathrm{~s}^{-1} \mathrm{Mpc}^{-1}$ (37). In addition, a fit of the late-time synthetic spectra to observations gave $H_{0}=68$ $\pm 13 \mathrm{~km} \mathrm{~s}^{-1} \mathrm{Mpc}^{-1}$ (38).

These estimated values tend to converge to $H_{0} \approx 58$ to $65 \mathrm{~km} \mathrm{~s}^{-1} \mathrm{Mpc}^{-1}$ where the error bars overlap. The higher $H_{0}$ is in conflict with the age of the universe for $\Omega_{\mathrm{M}}=1$ and $\Omega_{\Lambda}=0$, whereas the lower $H_{0}$ could avoid this problem. Further efforts to reduce the error bars will require the reexamination of sampling, extinction correc- tions, and so on.

Density parameter: In determining $\Omega_{\mathrm{M}}$ and $\Omega_{\Lambda}$ from SNe Ia, absolute calibration of the distances to SNe Ia is not needed, but more accurate measurements of magnitudes and $z$ values are necessary. In addition to the dispersion problem, whether a significant value of $\Omega_{M}$ can be obtained depends on (i) whether a suitable sample of remote SNe Ia can be obtained, and (ii) whether the peak luminosities of SNe Ia are sufficiently free from the effects of cosmic and galactic chemical evolutions.

More than 60 SNe Ia with high $z$ values have been observed $(39,40)$ (Fig. 5). Values derived from the first 7 supernovae at $z>0.35$ are $\Omega_{\mathrm{M}}=0.88_{-0.60}^{+0.69}$ for $\Omega_{\Lambda}=0$ or $\Omega_{\mathrm{M}}=1-\Omega_{\Lambda}=0.94_{-0.28}^{+0.34}$ for $\Omega_{\mathrm{M}}+\Omega_{\Lambda}=1$, assuming that evolutionary effects are small. To clarify whether the nature of high- $z \mathrm{SNe}$ Ia is the same as that of nearby $\mathrm{SNe}$ Ia, it is important to identify the progenitors' evolution and population (41). Systematic studies on the effects of metallicity of the progenitors are underway.

\section{REFERENCES AND NOTES}

1. A. V. Filippenko, Annu. Rev. Astron. Astrophys. (1997), in press; D. Branch, K. Nomoto, A. V. Filippenko, Comments Astrophys. 15, 221 (1991).

2. $\mathrm{Ch}$ is the maximum mass limit of the white dwarfs in hydrostatic equilibrium, with a typical value of 1.46 $M_{\odot}$. For larger masses, pressures exerted by degenerate electrons cannot sustain the gravity because of the special relativistic effect.

3. Thermonuclear Supernovae, P. Ruiz-Lapuente, R. Canal, J. Isern, Eds. (Kluwer,Dordrecht,1997); this book includes most of the recent progress in SNe Ia.

4. D. Branch and G. Tammann, Annu. Rev. Astron. Astrophys. 30, 359 (1992).

5. M. M. Phillips, Astrophys. J. 413, L105 (1993).

6. A. G. Riess, W. N. Press, R. P. Kirshner, Astrophys. J. 438, L17 (1995); Astrophys. J. 473, 88(1996).

7. D. Branch, M. Livio, L. R. Yungelson, F. R. Boffi, E. Baron, Publ. Astron. Soc. Pac. 107, 717(1995); A. Renzini, in IAU Colloquium 145, Supernovae and Supernova Remnants, R. McCray and Z. Wang, Eds.(Cambridge University Press,Cambridge,1996), pp.77-85.

8. I. Iben Jr. and A. Tutukov, Astrophys. J. Suppl. Ser. 55, 335(1984); R. Webbink, Astrophys. J. 277, 355 (1984).

9. For example, K. Nomoto, Astrophys. J. 253, 798 (1982); and Y. Kondo, Astrophys. J. 367, L19 (1991); K. Nomoto et al., in Supernovae (Les Houches, Session LIV) S. Bludman et al. Eds. (Elsevier Science,Amsterdam,1994), pp.199-249.

10. I. Hachisu, M. Kato, K. Nomoto, Astrophys. J. 470, 
L97 (1996).

11. K. Nomoto, Astrophys. J. 257, 780 (1982b); M. Hashimoto, K. Nomoto, K. Arai, K. Kaminisi, Astrophys. J. 307, 687 (1986).

12. S. E. Woosley and T. A. Weaver, Astrophys. J. 423, 371 (1994).

13. E. P. J. van den Heuvel, D. Bhattacharya, K. Nomoto, S. A. Rappaport, Astron. Astrophys. 262, 97 (1992); S.A. Rappaport, R. DiStefano, J. Smith, Astrophys. J. 426, 692 (1994).

14. P. Ruiz-Lapuente et al., Nature 365, 728 (1993).

15. W. D. Arnett, Nucleosynthesis and Supernovae (Princeton Univ. Press,Princeton,1996); S. E. Woosley, in Supernovae, A. Petschek Ed. (Springer,New York,1990), pp.182-212.

16. Fe peak elements consist of mostly $\mathrm{Fe}$ and $\mathrm{Ni}$ with some $\mathrm{Ti}, \mathrm{V}, \mathrm{Cr}, \mathrm{Mn}$, and Co.

17. For example, J. Niemeyer and W. Hillebrandt, $A s-$ trophys. J. 452, 769 (1995); J. Niemeyer and S. E. Woosley, Astrophys. J. 475, 740 (1997).

18. K. Nomoto, F. -K. Thielemann, K. Yokoi, Astrophys. J. 286, 644 (1984); F. -K. Thielemann, K. Nomoto, K. Yokoi, Astron. Astrophys. 158, 17 (1986); H. Yamaoka, T. Shigeyama, K. Nomoto, F.-K. Thielemann, Astrophys. J. 393, L55 (1992).

19. A. M. Khokhlov, Astron. Astrophys. 245, 114 (1991); S. E. Woosley and T. A. Weaver, in Supernovae (Les Houches,Session LIV), S. Bludman et al., Eds. (Elsevier Science,Amsterdam,1994), pp.63-154.

20. A. M. Khokhlov, Astron. Astrophys. 245, L25 (1991);

W. D. Arnett and E. Livne, Astrophys. J. 427, 314 (1994).

21. E. Livne, Astrophys. J. 354, L53 (1990).

22. T. Shigeyama, K. Nomoto, H. Yamaoka, F. -K. Thielemann, Astrophys. J. 386, L13 (1992); J. C. Wheeler and R. Harkness, Rep. Prog. Phys. 53, 1467 (1990).

23. P. Nugent, E. Baron, D. Branch, A. Fisher, P. Hauschildt, Astrophys. J. (1997), in press.

24. P. Höflich and A. Khokhlov, Astrophys. J. 457, 500 (1996).

25. For example, P. Mazzali, I.J. Danziger, M. Turatto, Astron. Astrophys. 297, 509 (1995); M. Turatto et al., Mon. Not. R. Astron. Soc. 283, 1 (1997).

26. P. Nugent, E. Baron, D. Branch, Astrophys. J. 455, L147 (1995).

27. For example, D. Jeffery et al., Astrophys. J. 397, 304 (1992); A. Fisher, D. Branch, P. Hoflich, A. Khokhlov, Astrophys. J. 447, L73 (1995); W. P. S. Meikle et al., Mon. Not. R. Astron. Soc. 281, 263 (1996).

28. P. Höflich et al., Astrophys. J. 472, L81 (1996).

29. M. Hashimoto, K. Nomoto, F. -K. Thielemann, Astron. Astrophys. 210, L5 (1989); F. -K. Thielemann, K. Nomoto, M. Hashimoto, Astrophys. J. 460, 408 (1996); T. Tsujimoto, K. Nomoto, Y. Yoshii, M. Hashimoto,
S. Yanagida, F. -K. Thielemann, Mon. Not. R. Astron. Soc. 277, 945 (1995); see also S. E. Woosley and T. A. Weaver, Astrophys. J. Suppl. Ser. 101, 181 (1995).

30. K. Nomoto et al., in (3), pp.349-378.

31. S. van den Bergh and G. A. Tammann, Annu. Rev. Astron. Astrophys. 29, 363 (1991).

32. For example, Y. Yoshii, T. Tsujimoto, K. Nomoto, Astrophys. J. 462, 266 (1996).

33. M. Hamuy et al., Astronomical J. 109, 1 (1995); Astronomical J. 112, 2398 (1996).

34. A. Sandage et al., Astrophys. J. 460, L15 (1996); A.

Saha et al., Astrophys. J. (1997) in press.

35. B. E. Schaefer, Astrophys. J. 460, L19 (1996).

36. W. D. Arnett, D. Branch, J. C. Wheeler, Nature 314, 337 (1985).

37. D. Branch, P. Nugent, A. Fisher, in (3), pp.715-734. 38. P. Ruiz-Lapuente, Astrophys. J. 465, L83 (1996).

39. The Supernova Cosmology Project: S. Perlmutter et al., Astrophys. J. 483 (1997), in press; IAU Circular 6596 (1997).

40. The High-Z Supernova Search Team: B. Schmidt et al., IAU Circular 6602 (1997).

41. P. Ruiz-Lapuente, A. Burkert, R. Canal, Astrophys. J. 447, L69 (1995); R. Canal, P. Ruiz-Lapuente, A. Burkert, Astrophys. J. 456, L101 (1996).

42. Supported in part by a grant-in-aid for scientific research $(05242102,06233101,4227)$ and COE research (07CE2002) of the Ministry of Education, Science, and Culture of Japan.

Figure 1: Spectra of SNe Ia (SN 1994D and SN 1990N) about 1 week before maximum brightness. SN1991T was peculiar (11). The y-axis is magnitude where the units of the flux $f_{\nu}$ are ergs $\mathrm{s}^{-1} \mathrm{~cm}^{-2} \mathrm{~Hz}^{-1}$.

Figure 2: Empirical family of the visual light curves of $\mathrm{SNe} \mathrm{Ia}$, which shows the brightness-decline rate relation. The triangles, squares, circles, and diamonds denote $\mathrm{SNe}$ 1991T, 1981B, 1986G, and 1991bg, respectively (6).

Figure 3: The non-LTE spectra of carbon deflagration model W7 (unmixed) at 20 days and 23 days past explosion, which are compared with SNe 1992A and 1994D, respectively (23). $\quad F_{\lambda}$ is the flux in units of ergs s $\mathrm{s}^{-1}$ Angstrom $^{-1}$.

Figure 4: Solar abundance pattern based on synthesized heavy elements from a composite of SNe Ia and SNe II with the most probable ratio (30). The dashed lines indicate typical uncertainties of a factor of 2 involved in the observational and theoretical abundances, where the abundance ratios are normalized to unity for ${ }^{56} \mathrm{Fe}$. The open and filled circles are used to clarify the association to elements.

Figure 5: Hubble diagram for the seven high- $z \mathrm{SNe}$ Ia out to $z \sim 0.6$, with some of the low- $z$ SNe Ia (33): (A) uncorrected blue magnitudes $m_{B}$, and (B) $m_{B}$ with corrections of the brightness-decline rate relation (39). 
The solid curves are theoretical $m_{B}$ values for $\left(\Omega_{\mathrm{M}}, \Omega_{\Lambda}\right)$ $=(0,0)$ (top curve), $(1,0)$ (middle curve) , and $(2,0)$ (bottom curve). The square points are not used in the analysis, because these points are corrected based on the extrapolation outside the range of light curve widths of low-redshift supernovae. 


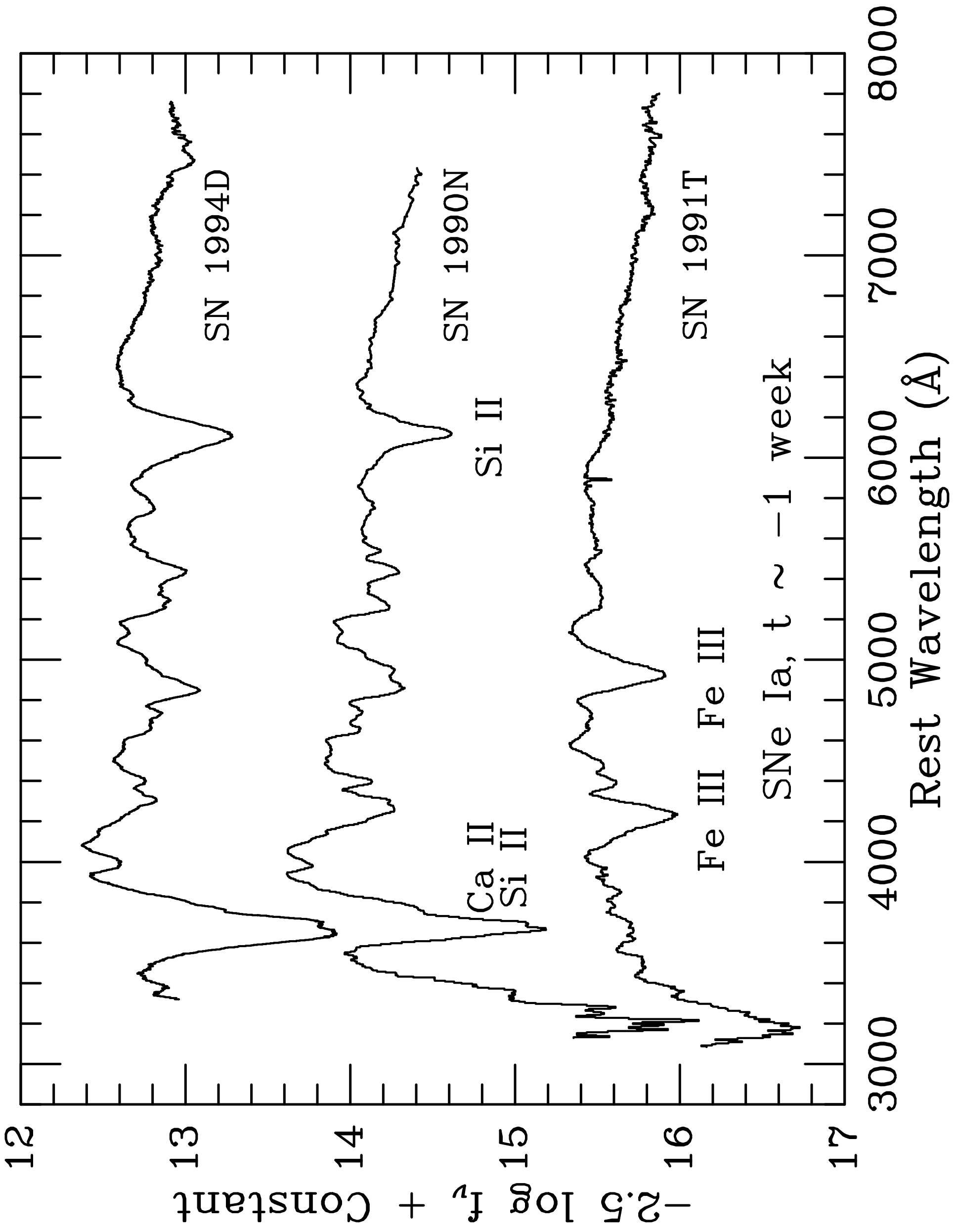




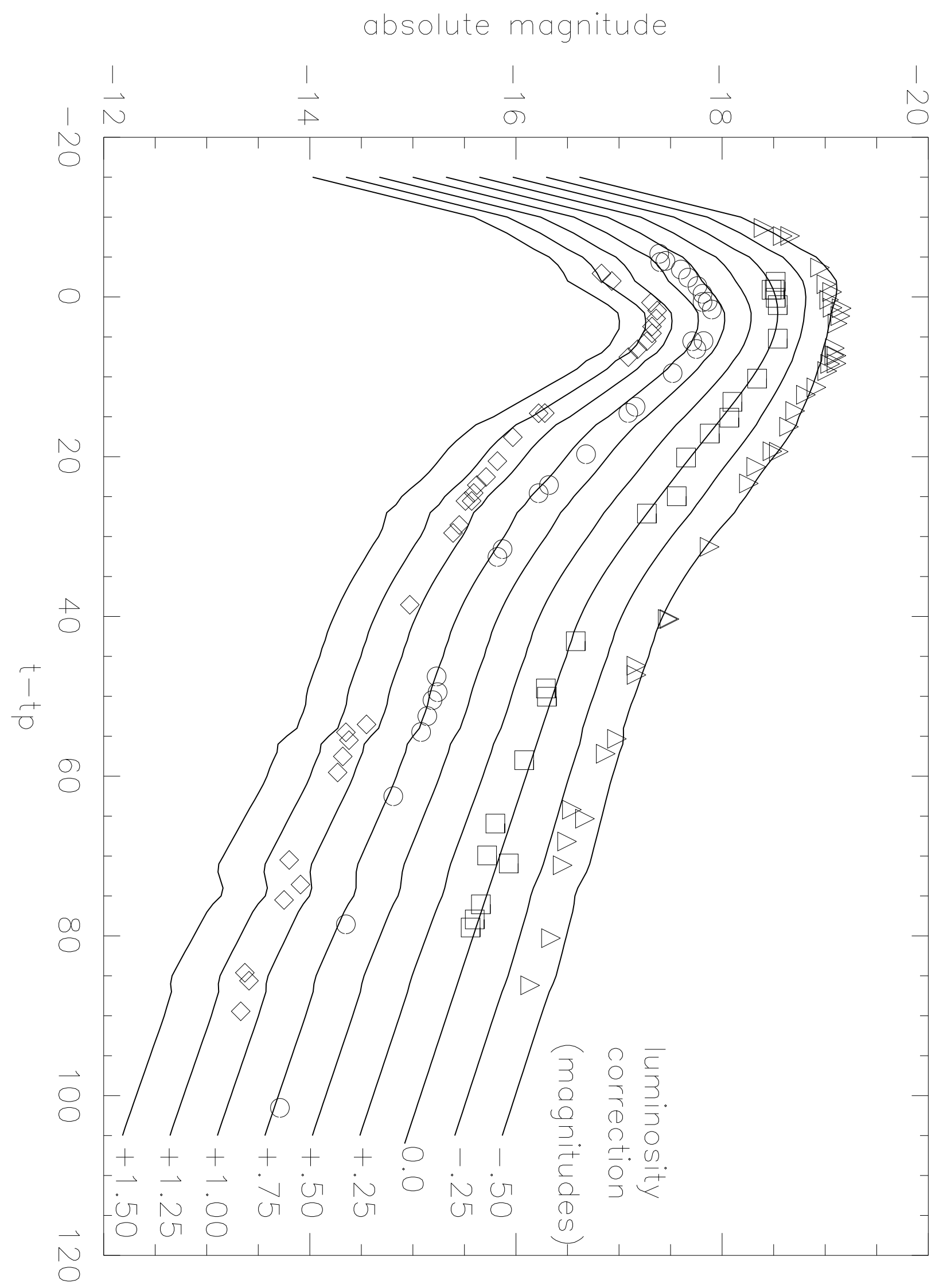




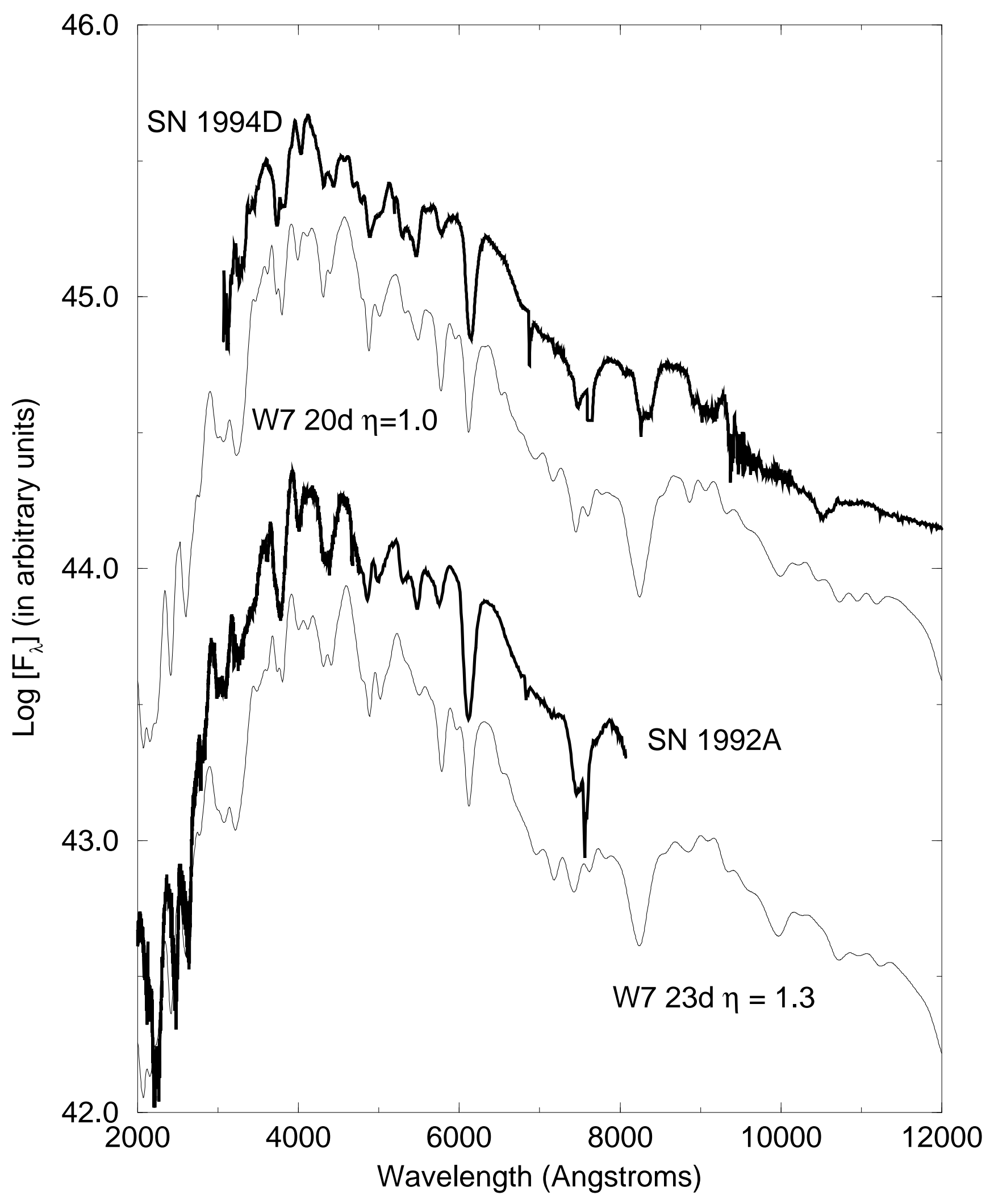




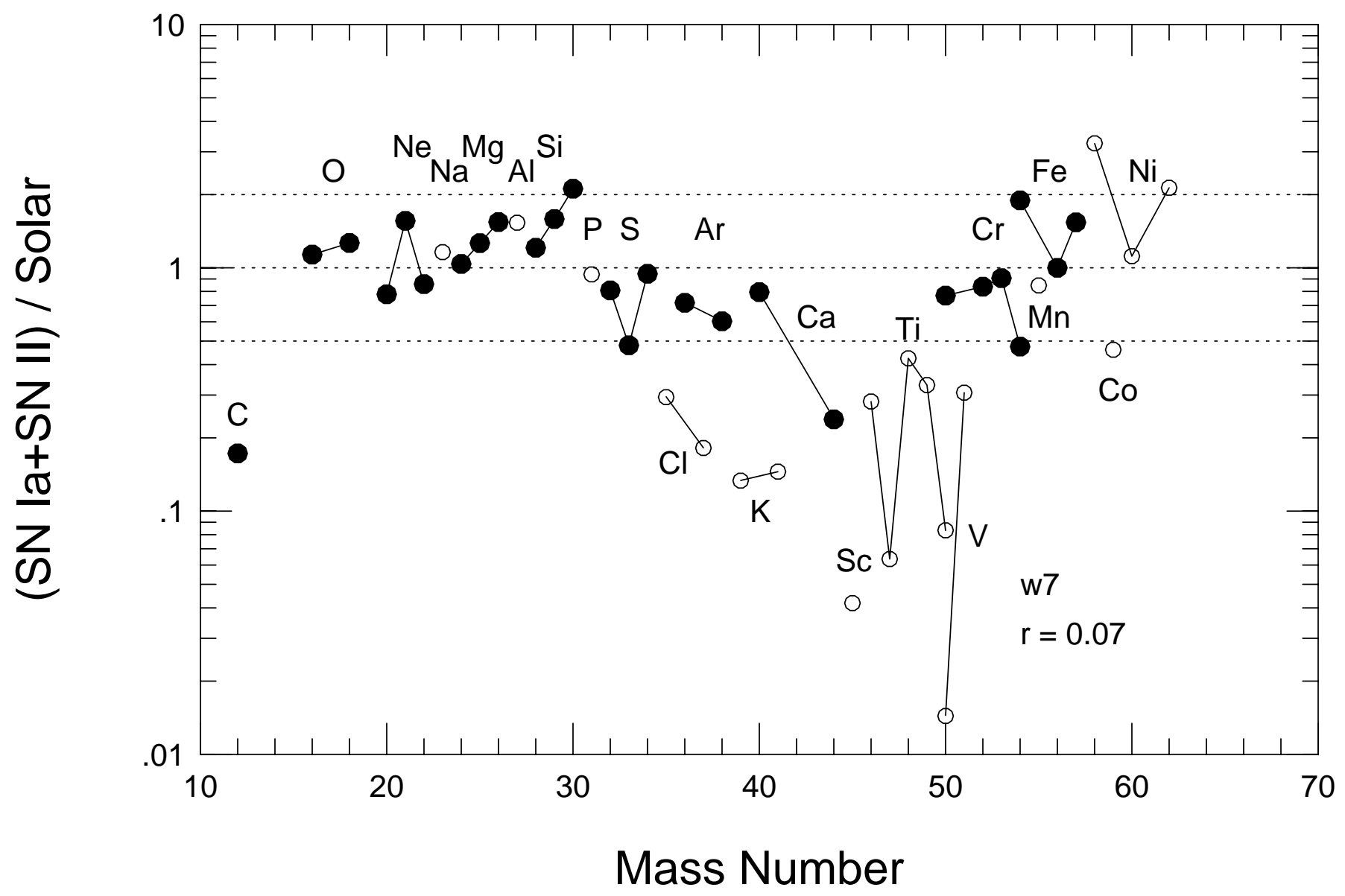




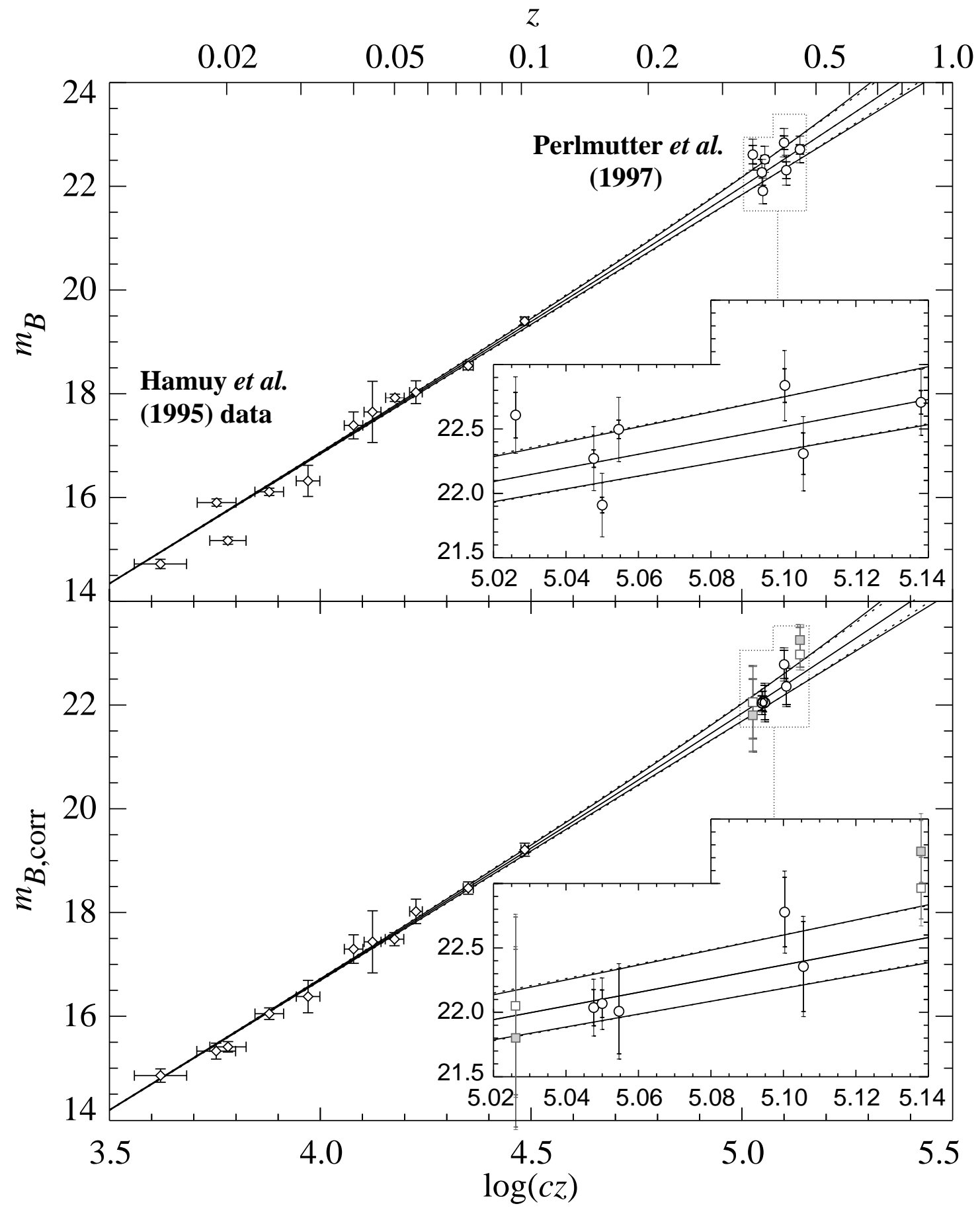

